$4^{\text {th }}$ Brazilian Conference on Composite Materials. Rio de Janeiro, July $22^{\text {nd }}-25^{\text {th }}, 2018$

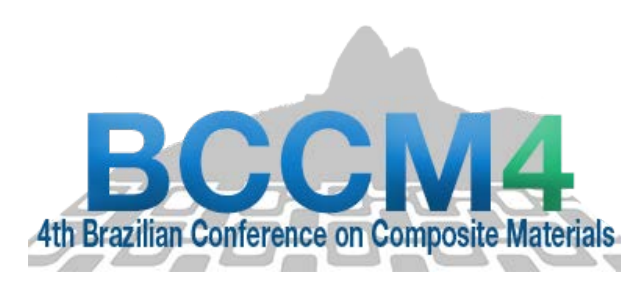

\title{
DEVELOPMENT AND CHARACTERIZATION OF CARBON FIBER REINFORCED THERMOPLASTICS - PART B: MECHANICAL PROPERTIES AND MICROSTRUCTURAL ANALYSIS
}

\author{
Bruno J. Lopes $\stackrel{(1)}{\text {, José Roberto M. d'Almeida }}{ }^{(1)}$
}

(1) Department of Chemical and Materials Engineering, Pontifícia Universidade Católica do Rio de Janeiro, Brazil

https://doi.org/10.21452/bccm4.2018.13.07

\begin{abstract}
Mechanical and microstructural properties of carbon fiber reinforced ABS (Acrylonitrile Butadiene Styrene) were analyzed and compared to neat, commercial ABS. Pellets of neat ABS and mixtures with varying fiber content (5\% and 16.7\%) and length (3 mm and $6 \mathrm{~mm}$ ) were submitted to mechanical testing and SEM (Scanning Electron Microscopy). Mechanical testing showed increases of up to $38 \%$ in tensile strength and $90 \%$ in modulus at the cost of loss of ductility. Surface analysis of pellets and fractured specimens carried out by SEM showed poor interface between fibers and matrix as well as a random distribution of the reinforcement phase along the composite.
\end{abstract}

Keywords: Composites; Carbon fiber; ABS; Extrusion, Additive Manufacturing.

\section{INTRODUCTION}

Much has been developed in the field of composite materials in the last decades. Researchers have found ways to make structural components faster and cheaper than ever before [1-4]. The development of thermoplastic matrix fiber reinforced composites broadens the range of applications and processing techniques for composite materials borrowing much from the knowhow developed for polymers.

The benefits of advanced fibers reinforced in common thermoplastics, such as ABS are very attractive. These benefits are especially attractive to novel processes like additive manufacturing, which usually rely on polymers as feedstock, limiting its breadth of ability. The change to fiber reinforced composite feedstock has been already proven to revolutionize the additive manufacturing industry [5-8]. The continued development of even more capable materials will only make this revolution come faster.

The objective of this paper was to evaluate the mechanical properties of a carbon fiber reinforced ABS thermoplastic and to observe its microstructure looking for characteristics that may influence the final properties of the composite. 


\section{EXPERIMENTAL}

\subsection{Background: Material processing}

For the initial material processing, pellets were produced mixing chopped carbon fiber (Tenax ${ }^{\circledR}-J$ HT C261, from Toho Tenax America, Inc.) in two different lengths (3 mm and $6 \mathrm{~mm}$ ) and ABS GP-35 (Terluran ${ }^{\circledR}$ GP-35, from INEOS Styrolution) at various weight percentages (\% wt). Each mixture was then extruded using a twin-screw extruder (Leistritz ZSE 18 MAXX) and chopped to produce pellets. For mechanical testing, filaments were extruded from each mixture's pellets using a micro extruder (Xplore Micro Compounder MC5).

More details regarding the processing stages, along with the calculation of the actual fiber content for each mixture can be seen in a previous work from the authors [9] and in Part A of this paper. Table 1 lists each mixture’s characteristics.

Table 1: Composition of each analyzed mixture including nominal and actual fiber content verified via TGA

\begin{tabular}{ccccc}
\hline Mixture & $\begin{array}{c}\text { Grape seed oil } \\
\% \text { wt }(\%)\end{array}$ & $\begin{array}{c}\text { Carbon Fiber } \\
\text { length (mm) }\end{array}$ & $\begin{array}{c}\text { Nominal \% wt CF } \\
(\%)\end{array}$ & $\begin{array}{c}\text { Actual \% wt CF } \\
(\%)\end{array}$ \\
\hline I & - & - & 0 & 0 \\
II & - & 3 & 5 & 3.1 \\
III & 1 & 3 & 5 & 5.2 \\
IV & 1 & 3 & 16.7 & 13.2 \\
V & 1 & 6 & 16.7 & 16.5 \\
\hline
\end{tabular}

\subsection{Mechanical Testing}

Mechanical testing was conducted to investigate properties such as tensile strength, Young's modulus and ductility. All tests were done using a universal testing machine (Oswaldo Filizola AME- $2 \mathrm{kN}$ ) with a constant rate of $50 \mathrm{~mm} / \mathrm{mm}$.

As extensometers were not used for these tests, Young's modulus values were obtained from the following equation, which takes into account the rigidity of the test rig:

$$
\frac{1}{K_{S}}=\frac{1}{K_{m}}+\frac{1}{E} \cdot \frac{L_{0}}{A_{0}}
$$

where $E$ is the Young's modulus, $L_{0}$ is the distance between grips and $A_{0}$ represents the test specimen's cross section area. $K_{m}$ is the rigidity of the test rig, determined by Novoa [10], to be 546.4 MPa. $K_{S}$ is related to the non-corrected Young's modulus value. This value can be achieved from a pair of coordinates extracted from the linear region of the load vs. deformation $(P \times \Delta l)$ plot.

\subsection{Microstructural Analysis}

Pellet cross section and the fracture surface of tested specimens were observed using a JEOL JSM-6510LV Scanning Electron Microscope (SEM) at $20 \mathrm{kV}$ and high vacuum. Samples were metallized with gold, via sputtering in a Balzers SCD 050 Sputter Coater.

Magnifications ranging from 100x to 5000x were used and are indicated in each image. 


\section{RESULTS AND DISCUSSION}

\subsection{Mechanical Properties}

The mechanical properties are shown in the box plots below, Figures 1(a) to (c). For each plot, the top and bottom of the box represent the 3rd and 1st quartiles of the data distribution, respectively, and the line in the middle of the box represents the median. The whiskers represent the maximum and minimum values within 1.5 interquartile range, whereas the crosshead marks represent the maximum and minimum values obtained. The square inside de boxes represents the mean of the data for each particular mixture.

Tensile strength, Figure 1(a), showed oscillating results. Initially, the introduction of carbon fiber to the ABS polymer (Mixture II) seemed to improve the tensile strength values reaching up to $14 \%$ increase. Mixture III, with the introduction of grape seed oil, showed a decrease back to neat ABS values. Since fiber content is very similar, the influence of the oil seems to be relevant to the final properties of the composite. Mixture IV, with a much greater \% wt of carbon fiber, showed a stark increase in tensile strength, reaching a maximum of $62.1 \mathrm{MPa}$ (38\% increase over neat ABS) but with large variance. This value is higher than what was found by Ning et al. [11, 12], who in their work used carbon fiber powder as reinforcement instead of fibers, but below what can be found in literature $[5,7,13]$.. Mixture $\mathrm{V}$, with the same \% wt of fibers, but with longer initial lengths, once again showed values similar to neat ABS. This oscillating behavior can be traced to poor fiber/matrix interface and also to the random distribution of the reinforcement along the composite, which will be investigated in more detail in subsequent item 3.2.

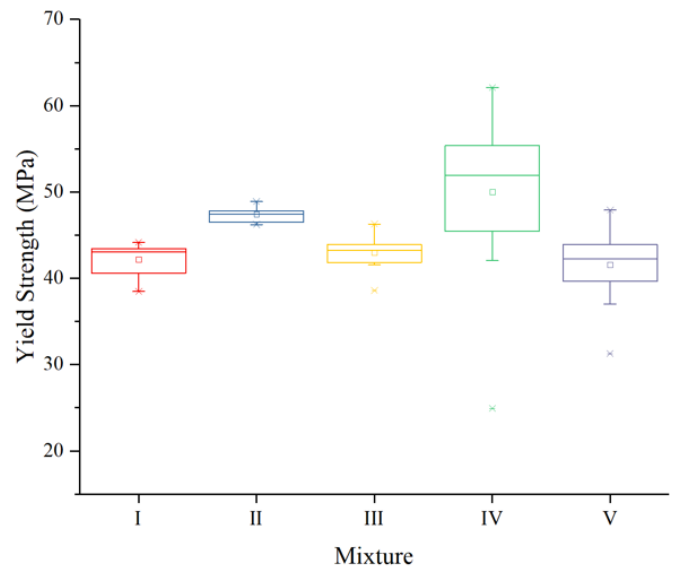

(a)

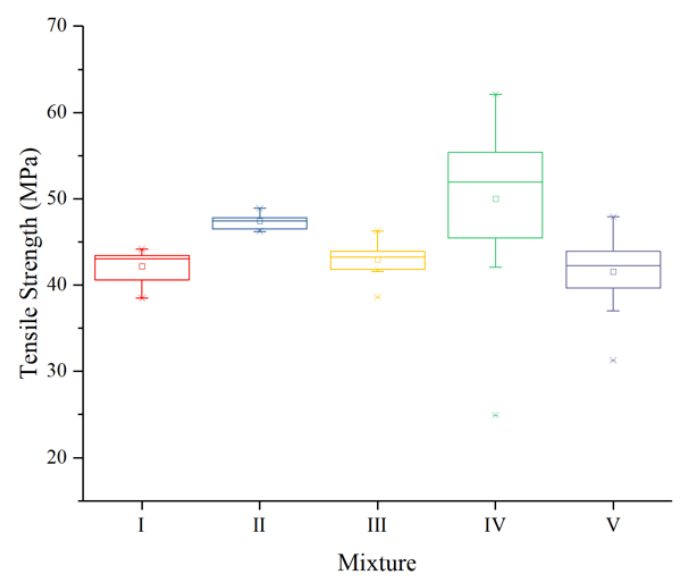

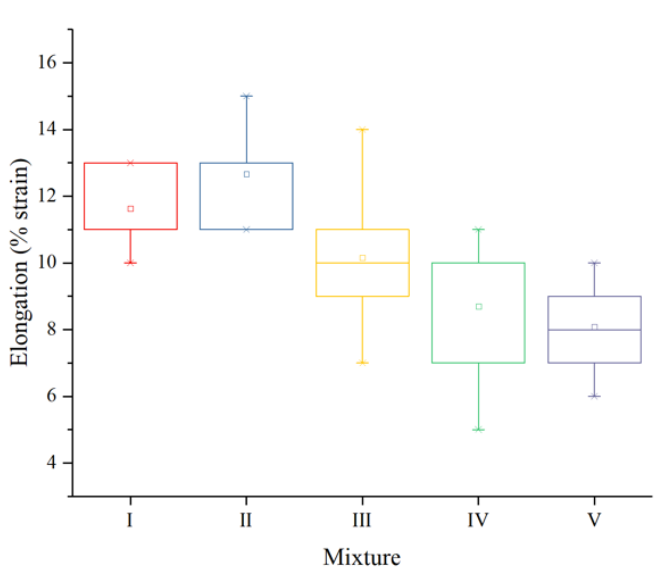

(b)

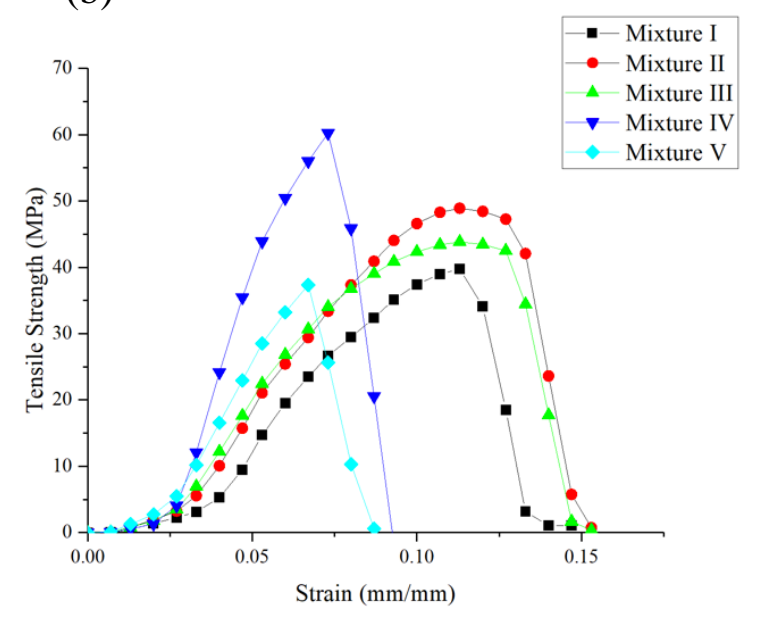


(c)

(d)

Figure 1: Box plot comparison of (a) Tensile strength; (b) Ductility (using elongation as a parameter); (c) Young's modulus; (d) A representative sample of each mixture during tensile testing

Ductility, Figure 1(b), showed a clear downward trend, as seen also in Ning et al. [12], despite large variations and small differences between each mixture. This is mostly due to the introduction of a high modulus and brittle material, such as carbon fiber, in the polymeric matrix. Greater \% wt of fibers caused a decrease between $25 \%$ and $30 \%$ over neat ABS.

Young's modulus, Figure 1(c), was the most affected property. Modulus increase followed the increase in carbon fiber content, reaching its peak at mixture IV, with a maximum of approximately 4.0 GPa (90\% increase over neat ABS). Once again, this value is higher than what was found by Ning et al. [12], but lower than some of the values that can be found in the literature [5, 7, 13]. Mixture V, with its longer initial carbon fiber length showed a decrease of 5\% over mixture IV, which was statistically relevant (t-Student test) despite relatively large variances.

Figure 1(d) shows the results of tensile testing for one sample from each mixture plotted together. The lower strains at rupture for mixtures with higher carbon fiber content are indicative of reduced ductility, showing a more brittle behavior.

\subsection{Microsctructure}

In the cross-section area of the pellets, Figures 2(a) and 2(b), it is possible to observe a large number of voids, as expected and seen in the literature [11]. With increasing \% wt of carbon fibers, more voids can be seen. These voids are formed during the cooling stages of the extrusion process by temperature gradients and are mostly constituted of entrapped air. The presence of voids is mainly due to sub-optimal process parameters, which were set to avoid matrix degradation [9].

In the fracture surface of tested specimens, Figures 2(c) and 2(d), the voids are completely eliminated. Due to the second extrusion process, the entrapped air was allowed to escape resulting in void-free microstructures. Also, it is possible to observe that the fracture initiation mechanism changed between neat ABS and reinforced samples. Neat ABS showed a very localized initiation site (indicated with an arrow), whereas reinforced samples had no clear initiation site. This difference confirms the transition from a more ductile behavior in neat ABS samples to more brittle in the carbon fiber/ABS mixtures, as observed in Figure 1(d).

In both the pellets and the fractured specimens, the interface between fibers and matrix is weak. Empty spaces can be seen around fibers, indicating poor adhesion. This can be further confirmed by the amount of holes left by pulled-off fibers. Higher magnifications show that the fibers do not damage the matrix while being pulled-off, thus reinforcing the poor adhesion observed. 


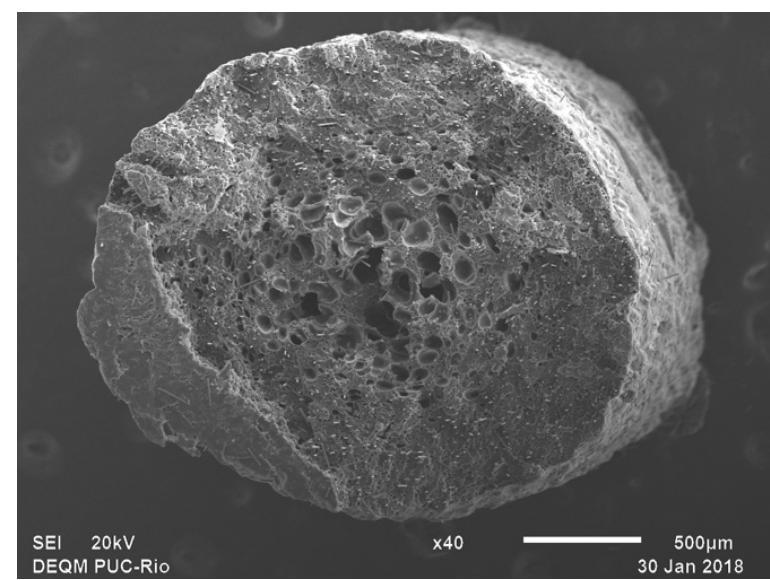

(a)

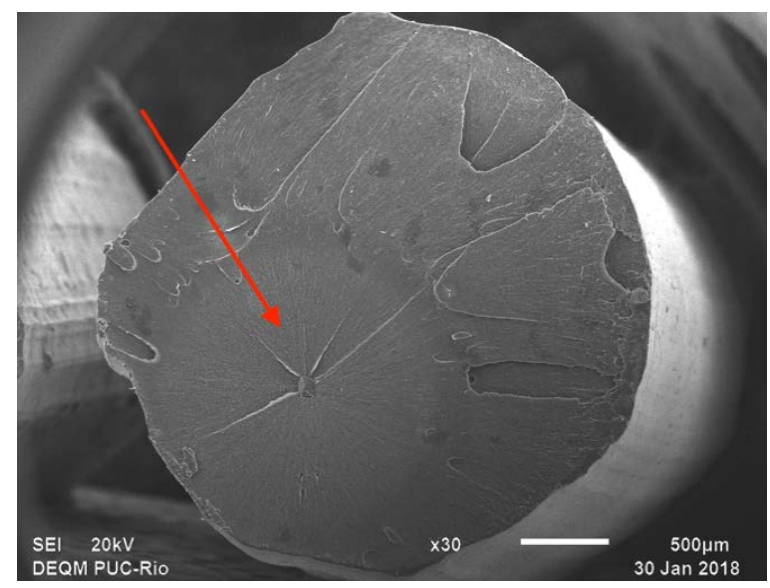

(c)

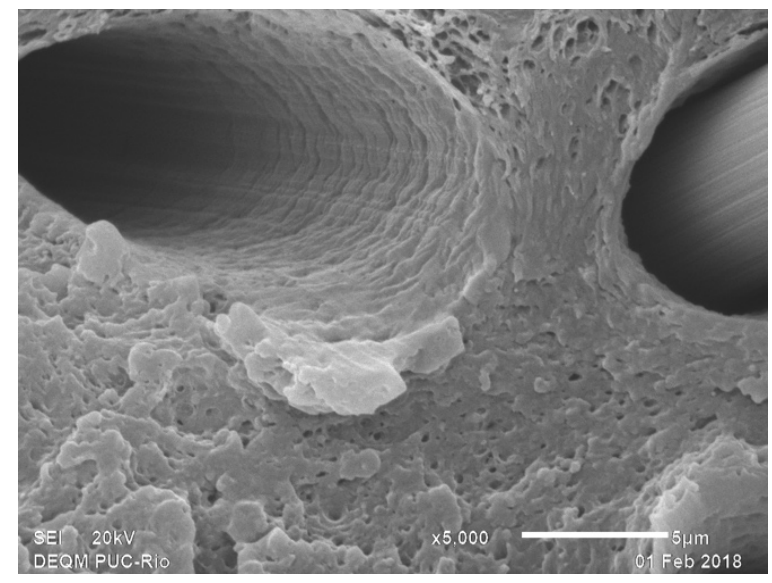

(e)

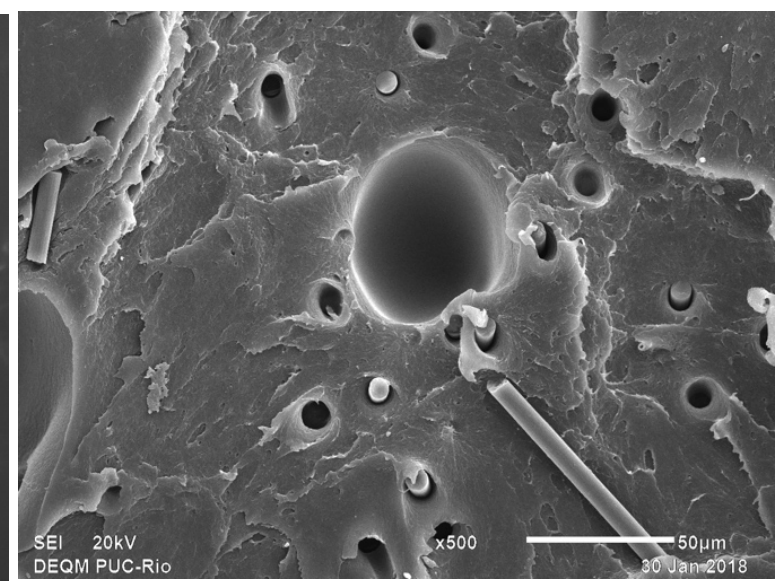

(b)

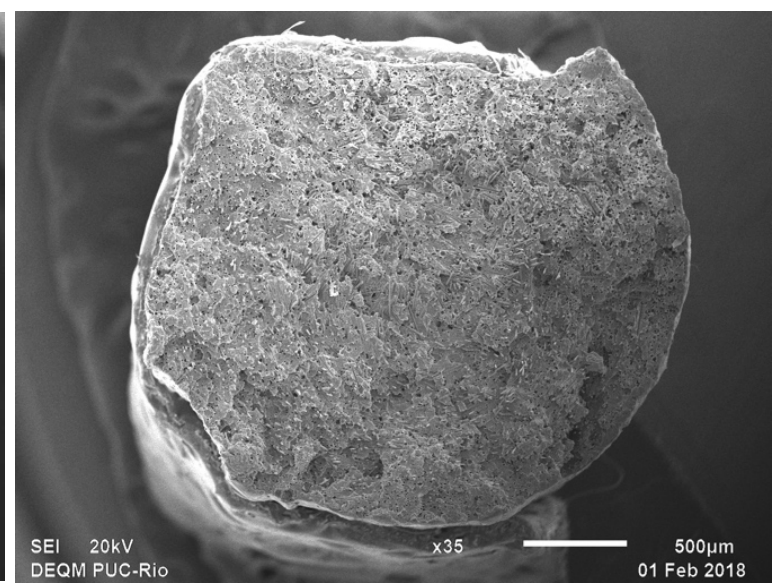

(d)

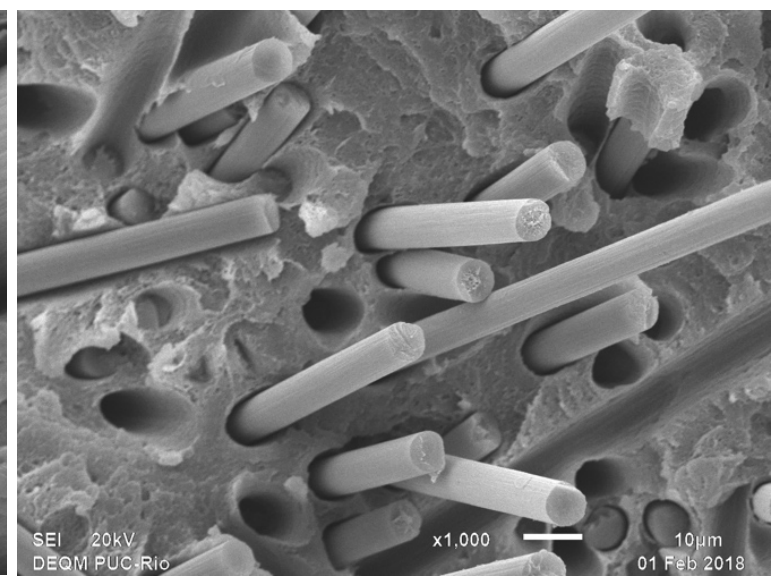

(f)

Figure 2: (a) Surface of a pellet (Mixture V); (b) Surface of a pellet at high magnification (Mixture II); (c) Surface of a fractured neat ABS (Mixture I) sample with an arrow indicating where failure begun; (d) Fractured surface of a sample with high \% wt CF (Mixture V); (e) Hole left from a pulled-off fiber and empty space around a fiber (Mixture V); (f) Random fiber distribution and interaction (Mixture V). 


\section{CONCLUSIONS}

The effects of the introduction of carbon fiber as reinforcement for neat ABS were successfully analyzed. Increases of up to 38\% in tensile strength and $90 \%$ in modulus were achieved over neat ABS. Although these results highlight the great potential of mechanical properties enhancement that carbon fiber can cause to for thermoplastics, the randomness of distribution and poor interface caused large variations in results, thus making it difficult to predict the actual structural performance of a final composite component. Furthermore, the effects of process additives, such as the grape seed oil, on the final characteristics of the composite should be more thoroughly investigated.

\section{ACKNOWLEDGEMENTS}

The authors acknowledge the support from the National Council for Scientific and Technologic Development (Conselho Nacional de Desenvolvimento Científico e Tecnológico - CNPq).

\section{REFERENCES}

[1] P. Feraboli, F. Gasco, B. Wade, S. Maier, R. Kwan, A. Masini, L. DeOto and M. Reggiani, 'Lamborghini Forged Composite technology for the suspension arms of the Sesto Elemento,’ in ASC Conference, Montreal, 2011.

[2] P. Feraboli, E. Peitso, T. Cleveland and P. B. Stickler, 'Modulus Measurement for Prepreg-based Discontinuous Carbon Fiber/Epoxy Systems,' Journal of Composite Materials, vol. 43, no. 19/2009, pp. 1947-1965, August 2009.

[3] P. Feraboli, E. Peitso, T. Cleveland, P. B. Stickler and J. C. Halpin, 'Notched behavior of prepreg-based discontinuous carbon fiber/epoxy systems,' Composites , vol. Part A, no. 40, pp. 289-299, March 2009.

[4] P. Feraboli, E. Peitso, F. Deleo, T. Cleveland and P. B. Stickler, 'Characterization of Prepreg-Based Discontinuous Carbon Fiber/Epoxy Systems,' Journal of Reinforced Plastics and Composites, vol. 28, no. 10/2009, pp. 1191-1214, May 2008.

[5] H. L. Tekinalp, V. Kunc, G. M. Velez-Garcia, C. E. Duty, L. J. Love, A. K. Naskar, C. A. Blue and S. Ozcan, 'Highly oriented carbon fiber-polymer composites via additive manufacturing,' Composites Science and Technology, vol. 105, pp. 144-150, 16 Oct 2014.

[6] L. J. Love and C. Duty, 'Cincinnati Big Area Additive Manufacturing (BAAM),' Oak Ridge, TN, 2015.

[7] L. J. Love, V. Kunc, O. Rios, C. E. Duty, A. M. Elliot, B. K. Post, R. J. Smith and C. A. Blue, 'The importance of carbon fiber to polymer additive manufacturing,' J. Mater. Res., vol. 29, no. 17, 14 Sep 2014.

[8] The Boeing Company, 'One For The Record Books,' 26 August 2016. [Online]. Available: $\quad$ http://www.boeing.com/features/2016/08/record-books-08-16.page. [Accessed 30 November 2016].

[9] B. J. Lopes, 'Desenvolvimento e Caracterização de Compósitos Termoplásticos Reforçados por Fibra de Carbono,’ PUC-Rio, Rio de Janeiro, 2018. 
[10] D. M. C. Novoa, 'Avaliação físico-química do polietileno de alta densidade processado pelo método de impressão 3D por modelagem por fusão e deposição FDM,' Rio de Janeiro, 2014.

[11] F. Ning, W. Cong, Z. Hu and K. Huang, 'Additive manufacturing of thermoplastic matrix composites using fused deposition modeling: A comparison of two reinforcements,' Journal of Composite Materials, vol. 51, no. 27, pp. 3733-3742, 1 November 2017.

[12] F. Ning, W. Cong, J. Wei, S. Wang and M. Zhang, 'Additive Manufacturing of CFRP Composites Using Fused Deposition Modeling: Effects of Carbon Fiber Content and Length,' in ASME 2015 International Manufacturing Science and Engineering Conference, Charlotte, North Carolina, 2015.

[13] C. E. Duty, V. Kunc, B. Compton, B. Post, D. Erdman and R. Smith, 'Structure and mechanical behavior of Big Area Additive Manufacturing (BAAM) materials,' Rapid Prototyping Journal, vol. 23, no. 1, pp. 181-189, January 2017. 